Artigo Original

Atenção à Saúde

\title{
CRESCIMENTO PONDERO-ESTATURAL DE PACIENTES DE 0 A 16 ANOS COM ANEMIA FERROPRIVA
}

\section{Weight and height growth of patients aged 0 to 16 years with iron deficiency anemia}

Cintia Vieira Niero ${ }^{1}$, Fábio Almeida Morais ${ }^{1}$, Kristian Madeira ${ }^{1}$, Guilherme Savi Serafim ${ }^{1}$, Anie Savi Serafim ${ }^{1}$, Priscyla Waleska Simões ${ }^{2}$

${ }^{1}$ Curso de Medicina - Universidade do Extremo Sul Catarinense - UNESC, Criciúma, SC, Brasil.

²Programa de Pós-Graduação em Saúde Coletiva (Mestrado Profissional) Universidade do Extremo Sul Catarinense - UNESC, Criciúma, SC, Brasil.

\section{Endereço para correspondência:}

Fabio Almeida de Morais

Curso de Medicina. Av. Universitária, 1105, Bairro Universitário. Criciúma - SC. CEP - 88806-000.

Email: fabicomorais@gmail.com 


\title{
Artigo Original \\ Atenção à Saúde
}

\section{Resumo}

Introdução: A anemia ferropriva figura entre os problemas de saúde mais comuns da infância, sendo um problema de saúde pública, uma vez que atinge em média $30 \%$ da população mundial. Objetivos: Avaliar a influência dos níveis de hemoglobina no crescimento pondero-estatural de pacientes com diagnóstico de anemia ferropriva de 0 a 16 anos. Métodos: Estudo observacional transversal, retrospectivo e quantitativo. A população foi composta por pacientes de 0 a 16 anos que realizaram duas ou mais consultas no período de janeiro/2010 a dezembro/2012, com o diagnóstico de anemia, resultando em 55 prontuários. Resultados: A idade mediana da população no momento do diagnóstico foi de 1,50 anos $(1,08-3,17)$. O grupo etário que obteve a maior frequência foi de 1 a 2 anos incompletos, totalizando $49,1 \% \quad(n=27)$ dos pacientes. A maior parcela dos pacientes fazia profilaxia com ferro, correspondendo a $50,9 \%(n=28)$. Quanto à classificação de peso dos pacientes para a idade em relação à hemoglobina, 3,6\% $(n=2)$ dos pacientes foram classificados como baixo peso, $76,4 \%$ ( $n=42)$ como eutróficos, $18,2 \%$ ( $n=10)$ como sobrepeso e um paciente $(1,8 \%)$ como peso elevado. Comparando-se a estatura para a idade dos pacientes em relação à hemoglobina, os eutróficos corresponderam a $89,1 \%(n=49)$ da amostra. Conclusão: Não houve relação entre déficit pondero-estatural e anemia ferropriva, porém parece haver relação entre a anemia ferropriva e tendência à obesidade.

Palavras-chave: Anemia ferropriva; Crescimento; Epidemiologia.

\begin{abstract}
Introduction: Iron deficiency anemia is among the most common health problems of childhood, being a public health problem, since it reaches on average $30 \%$ of the world population. To assess the influence of hemoglobin levels in growth weight and height of patients with iron deficiency anemia 0-16 years. Methods: Cross-sectional observational study, retrospective and quantitative. The population consisted of patients 0-16 years held two or more in the period January / 2010 to December / 2012, with a diagnosis of anemia, resulting in 55 records. Results: The median age of the population at diagnosis was 1.50 years (1.08 to 3.17 ). The age group had the highest frequency was 1 to 2 years of age, totaling $49.1 \%(n=27)$ of patients. The largest number of patients was prophylaxis with iron, corresponding to $50.9 \%(n=28)$. As for
\end{abstract}


patient weight rating for age in relation to hemoglobin, $3.6 \%(n=2)$ of patients were classified as underweight, $76.4 \%(n=42)$ as eutrophic, $18.2 \%(n=10)$ as overweight and one patient (1.8\%) and high weight. Comparing the height for age of patients in relation to hemoglobin, the eutrophic accounted for $89.1 \%(n=49)$ of the sample. Conclusion: There was no relationship between weight and height deficit and iron deficiency anemia, but there seems to be a relationship between iron deficiency anemia and tendency to obesity.

Keywords: Iron Deficiency Anemia; Growth; Epidemiology.

\section{INTRODUÇÃO}

A deficiência de ferro é a desordem nutricional mais comum e difundida em todo o mundo, e afeta um grande número de crianças e mulheres nos países nãodesenvolvidos, sendo a única deficiência de nutrientes que também possui significativa prevalência nos países industrializados. É estimado que a deficiência de ferro atinja quatro bilhões de indivíduos e que a anemia ferropriva acometa mais de dois bilhões de pessoas no mundo'.

A anemia ferropriva pode ser considerada como um estágio final de um longo período de balanço negativo do ferro ${ }^{2}$. Atualmente é reconhecido que não só a anemia, mas também a deficiência leve de ferro pode causar efeitos adversos no desenvolvimento cognitivo, na capacidade imunológica e na capacidade de trabalho.

A infância é um período crítico onde alterações no crescimento, como obesidade ou desnutrição, podem levar ao desenvolvimento de doenças crônicas em fases mais tardias. A avaliação do crescimento é a medida mais eficaz para avaliar a saúde e o estado nutricional de crianças, já que doenças e distúrbios nutricionais, independentemente de suas etiologias, afetam o crescimento infantil ${ }^{3}$. Constata-se um aumento exponencial de crianças menores de 5 anos com atraso de crescimento nos países em desenvolvimento. Como consequência ao crescimento restrito há um aumento do risco de contrair infecções, desenvolver um atraso cognitivo, além do risco aumentado de mortalidade ${ }^{4}$. O peso e a estatura são os dois índices mais importantes na avaliação do crescimento e pela aferição destes parâmetros podem ser calculados três índices antropométricos mais frequentemente empregados na avaliação 
nutricional: peso/idade, estatura/idade e peso/estatura ${ }^{3}$. Os gráficos de crescimento têm especial importância na avaliação sobre o crescimento, uma vez que com estes pode-se comparar a estatura e o peso encontrados com o que é esperado para crianças do mesmo sexo e idade ${ }^{5}$.

Devido à alta prevalência de anemia carencial em crianças, condição que cursa com alterações no desenvolvimento psicomotor a longo prazo, e por seus possíveis efeitos sobre o crescimento pondero-estatural, este estudo objetivou avaliar a influência dos níveis de hemoglobina no crescimento pondero-estatural de pacientes com diagnóstico de anemia ferropriva de 0 a 16 anos.

\section{MÉTODOS}

Foi realizado um estudo observacional, transversal, retrospectivo e de abordagem quantitativa.

Foram coletados de prontuários médicos dos pacientes acompanhados no Ambulatório de Pediatria da Universidade do Extremo Sul Catarinense, Criciúma - SC, no período de janeiro de 2010 a dezembro de 2012.

Foram incluídos na pesquisa os pacientes entre 0 e 16 anos que apresentaram duas ou mais consultas no período de janeiro de 2010 à dezembro de 2012, que possuíam o diagnóstico de anemia ferropriva e as informações necessárias [idade, sexo, peso, estatura, nível de hemoglobina, volume corpuscular médio (VCM), concentração de hemoglobina corpuscular média (CHCM), profilaxia com ferro e tempo de acompanhamento ambulatorial]. Foram excluídos os prontuários de crianças e adolescentes sem descrição de estatura, peso, hemoglobina, CHCM e VCM nos prontuários selecionados.

Inicialmente a amostra foi composta por 75 pacientes e, posteriormente, com a aplicação dos critérios de inclusão e exclusão, o número final foi de 55 prontuários, sendo excluídos os prontuários que não apresentaram as informações supracitadas necessárias ao diagnóstico da anemia ferropriva.

Os dados coletados foram organizados em planilhas do software IBM Statistical Package for the Social Sciencies (SPSS) versão 20.0. As análises estatísticas foram realizadas com um nível de significância $\alpha=0,05$ e um nível de confiança de $95 \%$. 
Os dados antropométricos obtidos (peso e estatura) foram analisados no programa Anthro versão 3.2.2 da OMS. O peso corporal foi determinado por meio de uma balança antropométrica digital graduada de 0 a $150 \mathrm{~kg}$, com resolução de 0,05 $\mathrm{kg}$, e a estatura, por meio de um estadiômetro portátil fixado à parede, graduado de 0 a $200 \mathrm{~cm}$, com escala de precisão de $0,2 \mathrm{~cm}$ para crianças acima de 2 anos. Crianças menores de 2 anos tiveram seu comprimento aferido em posição horizontal. O IMC foi calculado por meio do quociente da massa corporal em $\mathrm{kg}$ pela estatura $\mathrm{em}^{2}$.

Em relação à curva do percentil peso para idade, foram consideradas como baixo peso as crianças com percentil menor que 3 , eutróficas aquelas com percentil entre 3 e 85, sobrepeso (tendência à obesidade) com percentil maior que 85 até $97 \mathrm{e}$ peso elevado (obesidade) com percentil maior que 97 . Em relação à curva da percentil estatura para idade, foi considerado como baixa estatura aquelas com percentil menor que 3 , estatura adequada com percentil entre 3 e 97 e alta estatura maior que 0 percentil 97, de acordo com os critérios da OMS. O ponto de corte adotado no diagnóstico da anemia ferropriva foi de hemoglobina igual ou menor que $11 \mathrm{~g} / \mathrm{dL}$.

Para a investigação da diferença de média entre as variáveis hemoglobina e percentil do peso para a idade e entre hemoglobina e percentil da estatura para a idade foi realizada a aplicação do teste $U$ de Mann-Whitney para comparação de categorias com $n>2$.

Os dados coletados foram utilizados somente para pesquisa científica e foram garantidos o sigilo e identidade dos pacientes. O projeto foi aprovado pelo Comitê de Ética em Pesquisa da Universidade do Extremo Sul Catarinense (Projeto 225.025/2013).

\section{RESULTADOS}

Dos 75 prontuários/pacientes com registros/diagnóstico de anemia ferropriva, foram analisados 74 prontuários, pois $25,7 \%(n=19)$ não apresentavam todos os dados necessários e um não foi encontrado. Portanto, a amostra foi composta por 55 pacientes, com uma perda total de $26,7 \%(n=20)$ dos prontuários.

A idade mediana da população no momento do diagnóstico em anos foi de $1,50(1,08-3,17)$. O grupo etário que obteve a maior frequência foi de 1 a 2 anos 
Artigo Original

Atenção à Saúde

incompletos, totalizando $49,1 \%(n=27)$ dos pacientes. Na presente pesquisa não houve nenhum paciente diagnosticado entre 12 e 16 anos.

Tabela 1. Aspectos da Caracterização da Amostra $(n=55)$.

\begin{tabular}{cc}
\hline Variável & Mediana \pm AIQ ou n (\%) \\
\hline Idade (anos) & $9(16,4)$ \\
$0 \vdash 1$ & $27(49,1)$ \\
$1 \vdash 2$ & $5(9,1)$ \\
$2 \vdash 3$ & $3(5,5)$ \\
$3 \vdash 4$ & $5(9,1)$ \\
$4 \vdash 5$ & $3(5,5)$ \\
$5 \vdash 6$ & $1(1,8)$ \\
$7 \vdash 8$ & $1(1,8)$ \\
$8 \vdash 9$ & $1(1,8)$ \\
$11 \vdash 12$ & $1,5(1,08-3,17)$ \\
Idade no diagnóstico (anos) & $29(52,7)$ \\
Sexo & \\
Masculino & \\
\hline
\end{tabular}

$\mathrm{AIQ}$ = amplitude interquartílica

Em relação ao tempo de acompanhamento do paciente na Unidade Ambulatorial até o diagnóstico da doença, a mediana e a amplitude interquartílica foram de 258 (42 - 432) dias ou de 0,71 (0,12 - 1,18) anos.

Em relação ao sexo, a maior frequência correspondeu ao sexo masculino $(52,7 \% ; n=29)$ dos pacientes, embora não tenha havido diferença estatística, conforme ilustra a Tabela 1. A maior parcela dos pacientes fazia profilaxia com ferro, que correspondeu a $50,9 \%(n=28)$.

Os valores encontrados para os índices hematimétricos são descritos na Tabela 2. 
Tabela 2. Profilaxia com Ferro.

\begin{tabular}{cc}
\hline Variável & Média \pm DP ou n (\%) \\
\hline VCM (fl) & $73,35 \pm 8,95$ \\
CHCM $(\mathrm{g} / \mathrm{dL})$ & $32,14 \pm 1,43$ \\
Hemoglobina (g/dl & $10,19 \pm 0,75$ \\
Profilaxia com ferro & $28(50,9)$ \\
\hline DP = desvio padrão / VCM = volume corpuscular médio / CHCM = concentração de \\
hemoglobina corpuscular média (CHCM)
\end{tabular}

Observa-se valor de hemoglobina média de 10,02 g/dL para crianças menores de 2 anos, e 9 pacientes possuíam um nível de hemoglobina menor que $9,5 \mathrm{~g} / \mathrm{dL}$, que correspondeu a 16,36\% dos pacientes da pesquisa. Quanto a classificação de peso dos pacientes para a idade em relação à hemoglobina, 1 paciente $(1,8 \%)$ foi classificado como peso elevado, com um valor de hemoglobina de $8,7 \mathrm{~g} / \mathrm{dL}$, que correspondeu ao menor valor encontrado na amostra (Tabela 3).

Tabela 3. Classificação do Peso e Estatura X Hemoglobina.

\begin{tabular}{cccc}
\hline Variável & $\mathbf{n}(\%)$ & $\begin{array}{c}\text { Hemoglobina (g/dl) } \\
\text { (média } \pm \text { desvio padrão) }\end{array}$ & Valor - $\mathbf{p}^{*}$ \\
\hline $\begin{array}{c}\text { Classificação do } \\
\text { peso/ldade }\end{array}$ & $2(3,6)$ & $10,82 \pm 0,11$ & 0,683 \\
Baixo peso & $42(76,4)$ & $10,20 \pm 0,66$ & \\
Eutrófico & $10(18,2)$ & $10,15 \pm 1,01$ & \\
Sobrepeso & $1(1,8)$ & - & 0,813 \\
Peso elevado & & & \\
Classificação da & & $10,20 \pm 0,65$ & \\
estatura/ldade & $4(7,3)$ & $10,18 \pm 0,77$ & \\
Baixa estatura & $49(89,1)$ & $10,25 \pm 0,63$ & \\
Eutrófico & $2(3,6)$ & & \\
Alta estatura & ${ }^{*}$ teste U de Mann-Whitney para comparação apenas de categorias com $\mathrm{n}>2$.
\end{tabular}


Comparando-se a estatura para a idade dos pacientes em relação à hemoglobina, 7,3\% $(n=4)$ dos pacientes foram classificados com baixa estatura e obtiveram hemoglobina média de 10,20 \pm 0,65 $\mathrm{g} / \mathrm{dL}$, os eutróficos corresponderam a $89,1 \%(n=49)$ da amostra com hemoglobina média de 10,18 \pm 0,77 g/dL e 3,6\% ( $n=$ 2) pacientes foram classificados como tendo alta estatura com uma média de hemoglobina de 10,25 $\pm 0,63 \mathrm{~g} / \mathrm{dL}$.

\section{DISCUSSÃO}

Entre as doenças que acometem as crianças, a anemia ferropriva figura como uma das patologias mais prevalentes no período, por conta da situação nutricional e nível socioeconômico, em associação com o crescimento infantil6.

Este estudo encontrou um valor de hemoglobina média de 10,02 g/dL para crianças menores de 2 anos, valor maior do que o encontrado em um estudo realizado com crianças de creches públicas em Recife no ano de $1999^{7}$, onde o valor de hemoglobina média foi de $9,5 \mathrm{~g} / \mathrm{dl}$. Este valor pode refletir fatores como a curta duração do aleitamento materno exclusivo, a introdução do leite de vaca aliada a uma dieta pobre em ferro, bem como a maior predisposição às doenças infecciosas e parasitárias. Ambos os estudos não encontraram nenhum caso de anemia grave.

Um estudo realizado na cidade de Cascavel no ano de $2007^{8}$ com 256 crianças apontou um VCM de hemácias de 72,9 fl, condizente com o presente estudo, que mostrou um VCM médio de 73,35fl, demonstrando a carência de micronutrientes que ocorre associada ao déficit de ferro corporal. O mesmo trabalho também mostrou resultados relacionados ao sexo, onde 137 (53,5\%) dos indivíduos eram do sexo masculino e 119 (46,5\%) do sexo feminino, o que se encontra de acordo com os dados do nosso estudo, onde a maioria dos pacientes era do sexo masculino, embora não tenha havido diferença estatisticamente significativa. Um estudo realizado em Ribeirão Preto em $2005^{9}$ também obteve que $54,5 \%$ das crianças eram do sexo masculino.

O presente estudo mostrou que 9 pacientes possuíam um nível de hemoglobina menor que $9,5 \mathrm{~g} / \mathrm{dL}$, que correspondeu a $16,36 \%$ dos pacientes da pesquisa, dado que não está de acordo com o estudo realizado em Viçosa/MG ${ }^{10}$, que avaliou 205 crianças e mostrou que 41,5\% de crianças com anemia tinham 
hemoglobina menor que 9,5g/dL, fato corroborado com a intensidade da deficiência no grupo em questão consequente ao precoce esgotamento das reservas de ferro e longo período de duração da anemia.

O estudo realizado em Viçosa em $2003^{10}$ encontrou também que entre as crianças anêmicas, 40,9\% fazia uso de suplementos medicamentosos com ferro e, portanto, 60,1\% dos anêmicos não faziam tal uso, resultado concordante com a presente pesquisa, onde a maioria dos pacientes fazia uso de ferro. Tal fato pode estar refletindo problemas relacionados à frequência inadequada na ingestão e o uso de dose insuficiente do suplemento. Portanto, faz-se necessário o estudo das atuais práticas de suplementação, visando melhorar sua efetividade no controle da anemia. Embora a adoção de práticas alimentares adequadas contribua para a manutenção do adequado estado nutricional de ferro na infância, é indiscutível a importância de uma fonte adicional de ferro durante os 2 primeiros anos de vida, seja através da suplementação medicamentosa ou da fortificação de alimentos ${ }^{1}$.

O estudo realizado em Ribeirão Preto em $2005^{9}$ com crianças de 3 a 12 meses mostrou que a faixa etária com a maior prevalência de anemia foi entre 6 e 12 meses, fato concordante com o presente estudo, onde a faixa etária mais prevalente para a doença foi a faixa de 12 a 24 meses incompletos, totalizando 49,1\% dos pacientes. Tal fato refere-se possivelmente aos agravos nutricionais relacionados a esta faixa etária, como desmame precoce e introdução de alimentação com baixo teor de nutrientes.

A presente pesquisa associou o peso e estatura dos pacientes para a idade e obteve um resultado que mostrou que os eutróficos em relação ao peso corresponderam a 76,4\% dos indivíduos e 18,2\% dos pacientes se enquadraram na categoria sobrepeso, sendo que apenas um paciente (1,8\%) se enquadrou na categoria peso elevado e neste paciente foi encontrado o menor valor de hemoglobina do estudo, correspondendo a uma taxa de hemoglobina de $8,7 \mathrm{~g} / \mathrm{dL}$. Tal dado foi encontrado em um estudo realizado na cidade de Cascavel em $2007^{8}$, que observou uma prevalência de obesidade de 4,3\% entre as crianças com diagnóstico de anemia observadas, corroborando para uma prevalência de obesidade acima da média, o que indica a necessidade da adoção de medidas para aprimorar a oferta de nutrientes e um acompanhamento das crianças susceptíveis, para evitar que a desnutrição não reverta em outro problema de saúde pública - o sobrepeso. 
Dado que corrobora a tais achados foi encontrado no estudo realizado em Ilhabela ${ }^{11}$ no ano de 2007, que apresentou valores de sobrepeso em $13,6 \%$ das crianças participantes do estudo e obesidade em 6,8\% dos mesmos. O fato observado reflete as alterações alimentares presentes na dieta das crianças, correspondendo a dietas ricas em carboidratos e pobre em nutrientes como o ferro, o que contribui para um aumento da prevalência de sobrepeso e obesidade na infância.

Um estudo realizado em Maceió, no estado de Alagoas, no ano $2000^{12}$, analisou a prevalência de anemia em crianças da primeira série do ensino fundamental e mostrou que entre as crianças anêmicas não houve associação entre a anemia ferropriva e o retardo de crescimento estatural, dado que corrobora com o presente estudo, onde encontramos que a comparação de estatura para idade obteve $89,1 \%$ de indivíduos eutróficos, confirmando, portanto, dois achados importantes: as prevalências elevadas de anemia superam as prevalências de retardo ponderoestatural e a ausência de associação entre estes dois tipos de desnutrição se torna evidente.

Foi realizado um estudo em Xoxocotla, no México ${ }^{13}$, que avaliou os efeitos da suplementação com micronutrientes em crianças que receberam a suplementação com minerais como o ferro, e foi observado que a suplementação com micronutrientes esteve associada a um ganho estatural de aproximadamente $5 \mathrm{~mm}$ maior que o grupo placebo, comprovando que os micronutrientes são fatores importantes no crescimento pondero-estatural infantil.

Portanto, um grande preditor de nutrição adequada em crianças se refere a uma alimentação adequada e rica em nutrientes e minerais, onde o ferro participa de maneira única por ser de grande importância para a formação de hemoglobina. Ao ocorrer uma diminuição dos estoques de ferro do organismo, ocorre após certo período alterações do crescimento que podem ser traduzidas por déficit de estatura, desnutrição ou obesidade em graus variáveis.

Em nosso estudo não se observou uma relação entre déficit pondero-estatural e anemia ferropriva, portanto a importância da suplementação e acompanhamento da rotina alimentar torna-se de extrema importância para o reconhecimento e suspeita de anemia na infância, assim como a adoção de práticas alimentares que contenham nutrientes essenciais para o crescimento pondero-estatural infantil, a fim de evitar 0 surgimento de comorbidades que terão reflexos na vida adulta. 


\section{REFERÊNCIAS}

1. WHO. Iron deficiency anaemia: assessment, prevention and control: a guide for programme managers. World Health Organization. 2001.

2. Heijblom GS, Santos LMP. Anemia ferropriva em escolares da primeira série do ensino fundamental da rede pública de educação de uma região de Brasília, DF. Rev bras epidemiol. 2007;10(2):258-66.

3. Sousa FGM, Araújo TL. Avaliação nutricional de crianças de seis a sessenta meses. Rev Bras Enferm. 2004;57(5):534-6.

4. Lourenço BH, Villamor E, Augusto RA, Cardoso MA. Determinants of linear growth from infancy to school-aged years: a population-based follow-up study in urban Amazonian children. BMC public health. 2012;12(1):1.

5. Lopez FA, Junior DC. Tratado de Pediatria. Sociedade Brasileira de Pediatria. 1 ed. Barueri: Manole; 2007.

6. Braga JAP, Vitalle MSS. Deficiência de ferro na criança. Rev bras hematol hemoter. 2010;32(supl 2):38-44.

7. Vieira ACF, Diniz AS, Cabral PC, Oliveira RS, Lóla MMF, Silva SMM, et al. Avaliação do estado nutricional de ferro e anemia em crianças menores de 5 anos de creches públicas. J pediatr. 2007;83(4):370-6.

8. Rodrigues VC, Mendes BD, Gozzi A, Sandrini F, Santana RG, Matioli G. Deficiência de ferro, prevalência de anemia e fatores associados em crianças de creches públicas do oeste do Paraná, Brasil. Rev nutr. 2011;24(3):407-20.

9. Reis MCG, Nakano AMS, Silva IA, Gomes FA, Pereira MJB. Prevalência de anemia em crianças de 3 a 12 meses de vida em um serviço de saúde de Ribeirão Preto, SP, Brasil. Rev lat am enfermagem. 2010;18(4):792-9.

10. Silva DG, Franceschini SCC. Fatores de risco para anemia em lactentes atendidos nos serviços públicos de saúde: a importância das práticas alimentares e da suplementação com ferro. J Pediatr. 2007;83(2):149-56.

11. Costa JT, Bracco MM, Gomes PAP, Gurgel RQ. Prevalence of anemia among preschoolers and response to iron supplementation. J pediatr. 2011;87(1):76-9.

12. Santos CD, Santos LMP, Figueiroa JN, Marroquim PMG, Oliveira MAA. Anemia em escolares da primeira série do ensino fundamental da rede pública de Maceió, Alagoas, Brasil. Cad saúde pública. 2002;18:1757-63. 
Artigo Original

Atenção à Saúde

13. Rivera JA, Cossío TG, Flores M, Romero M, Rivera M, Rojo MMT, et al. Multiple micronutrient supplementation increases the growth of Mexican infants. Am j clin nutr. 2001;74(5):657-63. 